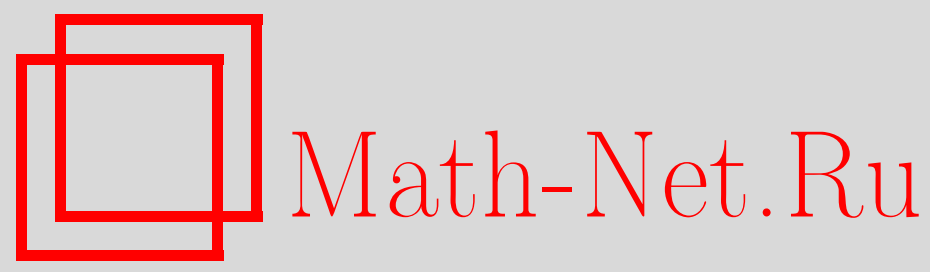

Л. И. Данилов, Мерозначные почти периодические функции, Матем. заметки, 1997, том 61, выпуск 1, 57-68

DOI: https://doi.org/10.4213/mzm1482

Использование Общероссийского математического портала Math-Net.Ru подразумевает, что вы прочитали и согласны с пользовательским соглашением http://www. mathnet.ru/rus/agreement

Параметры загрузки:

IP: 54.162 .127 .20

26 апреля 2023 г., 12:47:08

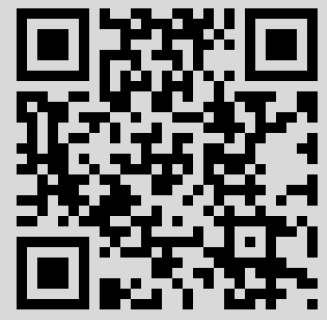




\title{
МЕРОЗНАЧНЫЕ ПОЧТИ ПЕРИОДИЧЕСКИЕ ФУНКЦИИ
}

\author{
Л.И. Данилов
}

Рассматриваются почти периодические по Степанову функции $\mu \in S(\mathbb{R}, \mathscr{M})$ со значениями в метрическом пространстве вероятностных борелевских мер $\mathscr{M}$ с метрикой Прохорова, определенных на полном сепарабельном метрическом пространстве $\mathscr{U}$. Основной результат: функция $t \rightarrow \mu[\cdot ; t] \in \mathscr{M}, t \in \mathbb{R}$, принадлежит пространству $S(\mathbb{R}, \mathscr{M})$ тогда и только тогда, когда для всех ограниченных непрерывных функций $\mathscr{F} \in C_{b}(\mathscr{U}, \mathbb{R})$ функции $\int_{\mathscr{U}} \mathscr{F}(x) \mu[d x ; \cdot]$ почти периодические по Степанову (степени 1) при этом

$$
\operatorname{Mod} \mu=\sum_{\mathscr{F} \in C_{b}(\mathscr{U}, \mathbb{R})} \operatorname{Mod} \int_{\mathscr{U}} \mathscr{F}(x) \mu[d x ; \cdot] .
$$

Библиографория: 17 названий.

В работе рассматриваются почти периодические (п.п.) по Степанову функции со значениями в пространстве вероятностных борелевских мер $\mathscr{M}(\mathscr{U})$, определенных на полном сепарабельном метрическом пространстве $\mathcal{U}$.

Мерозначные функции естественно возникают в задачах теории управления [1]-[3]. Ряд свойств п.п. по Степанову мерозначных функций приведен в [4]. Для задач оптимального п.п. управления они применялись в [5], [6]. Мерозначные функции используются также при исследовании вопроса о п.п. сечениях многозначных отображений. В частности, при характеристике многозначных отображений $t \rightarrow F(t), t \in \mathbb{R}$, с замкнутьми образами (в полном сепарабельном метрическом пространстве $\mathscr{U}$ ), которые при почти всех (п.в.) $t \in \mathbb{R}$ представимы в виде замыкания множества $\bigcup_{j} f_{j}(t)$, где $f_{j} \in S(\mathbb{R}, \mathscr{U})-$ п.п. по Степанову функции. Все такие многозначные отображения и только они являются носителями $\operatorname{supp} \mu[\cdot ; \cdot]$ мерозначных п.п. по Степанову функций $t \rightarrow \mu[\cdot ; t] \in \mathscr{M}(\mathscr{U})$, $t \in \mathbb{R}$ (для компактных метрических пространств $\mathscr{U}$ см. [7], [8]). Ряд утверждений о п.п. сечениях многозначных отображений $t \rightarrow \operatorname{supp} \mu[\cdot ; t]$, свойства которых определяются мерозначной п.п. по Степанову функцией $t \rightarrow \mu[\cdot ; t] \in \mathscr{M}(\mathcal{U})$, содержатся в [9], [10].

В п. 1 приводятся определения и некоторые стандартные утверждения о п.п. функциях, а также о вероятностных борелевских мерах, которые используются в дальнейшем. Большинство утверждений о п.п. функциях можно найти в [11], [12] (теорема Фреше [13] позволяет непосредственно переносить многие из них с нормированного пространствана метрическое). Относительно утверждений о вероятностных борелевских мерах см. [14]. Основные результаты работы приведены в п. 2. В п. 3 доказывается теорема 1, сформулированная в предыдущем пункте.

1. Пусть $(\mathscr{U}, \rho)$ - полное сепарабельное метрическое пространство. Через $\bar{A}$ обозначается замыкание множества $A \subset \mathscr{U}, K(x, r)=\{y \in \mathscr{U}: \rho(x, y) \leqslant r\}, x \in \mathscr{U}, r \geqslant 0$. Множество $K \subset \mathscr{U}$ предкомпактно, если $\bar{K}$ - компактное множество. 
Если $T_{j} \subset \mathbb{R}, j \in \mathbb{N},-$ измеримые (по Лебегу) множества, $T_{i} \cap T_{j}=\varnothing$ при $i \neq j$, $\operatorname{mes}\left(\mathbb{R} \backslash \bigcup_{j} T_{j}\right)=0$ (mes-мера Лебега) и $x_{j} \in \mathscr{U}$, то через $\sum x_{j} \chi_{T_{j}}(\cdot)$, где $\chi_{T_{j}}-$ характеристические функции множеств $T_{j}$, обозначается элементарная функция [14], определенная на множестве $\bigcup_{j} T_{j}$ и принимающая при $t \in T_{j}$ значение $x_{j}, j \in \mathbb{N}$. Такое же обозначение используется и для конечного числа множеств $T_{j}$, если mes $\left(\mathbb{R} \backslash \bigcup_{j} T_{j}\right)=0$. Функция $f: \mathbb{R} \rightarrow \mathscr{U}$ измерима [14], если для любого $\varepsilon>0$ существует элементарная функция $\sum x_{j} \chi_{T_{j}}(\cdot)$ такая, что

$$
\underset{t \in \mathbb{R}}{\operatorname{essup}} \rho\left(f(t), \sum x_{j} \chi_{T_{j}}(t)\right)<\varepsilon .
$$

Если $f_{j}: \mathbb{R} \rightarrow \mathscr{U}, j \in \mathbb{N},-$ измеримые функции, то аналогично элементарным функциям введем обозначение $\sum f_{j}(\cdot) \chi_{T_{j}}(\cdot)$ для измеримой функции, совпадающей с функцией $f_{j}(t)$ при $t \in T_{j} \subset \mathbb{R}, j \in \mathbb{N}$.

Пусть $M(\mathbb{R}, \mathscr{U})$ - класс измеримых функций $f: \mathbb{R} \rightarrow \mathscr{U}$, при этом функции, совпадающие при п.в. $t \in \mathbb{R}$, отож дествляются. Фиксируем элемент $x_{0} \in \mathscr{U} ; M_{1}(\mathbb{R}, \mathscr{U})$ - класс функций $f \in M(\mathbb{R}, \mathscr{U})$, для которых

$$
\sup _{\xi \in \mathbb{R}} \int_{\xi}^{\xi+1} \rho\left(f(t), x_{0}\right) d t<+\infty .
$$

Для функций $f, g \in M_{1}(\mathbb{R}, \mathscr{U})$ положим

$$
D_{1}^{(\rho)}(f, g)=\sup _{\xi \in \mathbb{R}} \int_{\xi}^{\xi+1} \rho(f(t), g(t)) d t .
$$

Множество почти периодических по Степанову функций $f: \mathbb{R} \rightarrow \mathscr{U}$ степени 1 обозначается через $S_{1}(\mathbb{R}, \mathscr{U})$. Функция $f: \mathbb{R} \rightarrow \mathscr{U}$ принадлежит $S_{1}(\mathbb{R}, \mathscr{U})$ тогда и только тогда, когда $f \in M_{1}(\mathbb{R}, \mathscr{U})$ и для любого $\varepsilon>0$ множество чисел $\tau \in \mathbb{R}$, для которых $D_{1}^{(\rho)}(f(\cdot+\tau), f(\cdot))<\varepsilon$, относительно плотно [11] (множество $\mathscr{E} \subset \mathbb{R}$ относительно плотно, если существует число $l>0$ такое, что $\mathscr{E} \cap[\xi, \xi+l] \neq \varnothing$ для всех $\xi \in \mathbb{R})$. Последовательность $\left\{\tau_{j}\right\} \subset \mathbb{R}$ назьвается $f$-возвращающей для функиии $f \in S_{1}(\mathbb{R}, \mathscr{U})$, если $D_{1}^{(\rho)}\left(f\left(\cdot+\tau_{j}\right), f(\cdot)\right) \rightarrow 0$ при $j \rightarrow+\infty$. Для каждой функции $f \in S_{1}(\mathbb{R}, \mathscr{U})$ определяется множество $\operatorname{Mod} f$, состоящее из тех чисел $\lambda \in \mathbb{R}$, для которых $e^{i \lambda \tau_{j}} \rightarrow 1$ при $j \rightarrow+\infty$ для любой $f$-возвращающей последовательности $\left\{\tau_{j}\right\}$. Наоборот, если $\left\{\tau_{j}\right\} \subset \mathbb{R}$ и $e^{i \lambda \tau_{j}} \rightarrow 1$ при $j \rightarrow+\infty$ для любого числа $\lambda \in \operatorname{Mod} f$, то $\left\{\tau_{j}\right\}-f$-возвращающая последовательность. Для любой функции $f \in S_{1}(\mathbb{R}, \mathscr{U})$, которая не совпадает (п.в.) с постоянной, множество $\operatorname{Mod} f$ счетное. Если $\mathscr{U}$ - банахово пространство, то $\operatorname{Mod} f$ совпадает с модулем показателей Фурье функции $f \in S_{1}(\mathbb{R}, \mathscr{U})$.

Пусть $f \in M_{1}(\mathbb{R}, \mathscr{U}), f_{j} \in S_{1}(\mathbb{R}, \mathscr{U}), j \in \mathbb{N}$, и $D_{1}^{(\rho)}\left(f, f_{j}\right) \rightarrow 0$ при $j \rightarrow+\infty$. Тогда $f \in S_{1}(\mathbb{R}, \mathscr{U})$ и $\operatorname{Mod} f \subset \sum \operatorname{Mod} f_{j}$ (сумма модулей определяется как наименьший модуль (группа по сложению), содержаший все слагаемые).

$\Phi$ ункция $f \in M(\mathbb{R}, \mathscr{U})$ принадлежит пространству $S(\mathbb{R}, \mathscr{U})$ почти периодических по Степанову функций, если для любых $\varepsilon, \delta>0$ множество тех чисел $\tau \in \mathbb{R}$, для которых

$$
\sup _{\xi \in \mathbb{R}} \operatorname{mes}\{t \in[\xi, \xi+1]: \rho(f(t+\tau), f(t)) \geqslant \varepsilon\}<\delta,
$$

относительно плотно. 
Определим функцию $\theta: \theta(\xi)=0$ при $\xi \leqslant 0, \theta(\xi)=\xi$ при $0 \leqslant \xi \leqslant 1$ и $\theta(\xi)=1$ при $\xi \geqslant 1$. Введем метрику $\rho^{\prime}(x, y)=\theta(\rho(x, y)), x, y \in \mathscr{U}$. Функция $f: \mathbb{R} \rightarrow \mathscr{U}$ принадлежит пространству $S(\mathbb{R}, \mathscr{U})$ тогда и только тогда, когда $f \in S_{1}\left(\mathbb{R},\left(\mathscr{U}, \rho^{\prime}\right)\right)(\operatorname{Mod} f$ для функции $f \in S(\mathbb{R}, \mathscr{U})$ определяется как для функции из пространства $\left.S_{1}\left(\mathbb{R},\left(\mathscr{U}, \rho^{\prime}\right)\right)\right)$. Для функций $f, g \in M(\mathbb{R}, \mathscr{U})$ положим $D^{(\rho)}(f, g)=D_{1}^{\left(\rho^{\prime}\right)}(f, g)$. Множество $S(\mathbb{R}, \mathscr{U})$ замкнуто в полном метрическом пространстве $\left(M(\mathbb{R}, \mathscr{U}), D^{(\rho)}\right)$. Если $f \in S_{1}(\mathbb{R}, \mathscr{U})$, то $f \in S(\mathbb{R}, \mathscr{U})$ и $\operatorname{Mod} f$ не зависит от рассматриваемого пространства функций.

Далее будем использовать краткие обозначения $S_{1}=S_{1}(\mathbb{R}, \mathbb{R})$ и $S=S(\mathbb{R}, \mathbb{R})$. Пусть $S(\mathbb{R})$ - совокупность измеримых множеств $T \subset \mathbb{R}$ таких, что $\chi_{T}(\cdot) \in S_{1}$. Для множеств $T \in S(\mathbb{R})$ положим $\operatorname{Mod} T=\operatorname{Mod} \chi_{T}(\cdot)$. Если $T \in S(\mathbb{R})$, то $\mathbb{R} \backslash T \in S(\mathbb{R})$ и $\operatorname{Mod}(\mathbb{R} \backslash T)=\operatorname{Mod} T$. Если $T_{1}, T_{2} \in S(\mathbb{R})$, то $T_{1} \cap T_{2} \in S(\mathbb{R})$ и $\operatorname{Mod} T_{1} \cap T_{2} \subset$ $\operatorname{Mod} T_{1}+\operatorname{Mod} T_{2}$. Для любого измеримого множества $T \subset \mathbb{R}$ положим

$$
\varkappa(T)=\sup _{\xi \in \mathbb{R}} \operatorname{mes}([\xi, \xi+1] \backslash T) .
$$

Если $T_{j} \in S(\mathbb{R}), j \in \mathbb{N}$, и $\sum_{j} \varkappa\left(T_{j}\right)<+\infty$, то $\bigcap T_{j} \in S(\mathbb{R}), \operatorname{Mod} \bigcap T_{j} \subset \sum_{j} \operatorname{Mod} T_{j}$ и $\varkappa\left(\bigcap T_{j}\right) \leqslant \sum_{j} \varkappa\left(T_{j}\right)$.

Пусть $\Lambda$ - произвольный модуль (группа по сложению) в $\mathbb{R}$. Обозначим через $\mathfrak{M}(\Lambda)$ множество последовательностей $\left\{T_{j}\right\}, j \in \mathbb{N}$, непересекающихся множеств $T_{j} \in S(\mathbb{R})$ таких, что $\operatorname{Mod} T_{j} \subset \Lambda$ и

$$
\lim _{N \rightarrow+\infty} \varkappa\left(\bigcup_{j \leqslant N} T_{j}\right)=0
$$

(последовательности из $\mathfrak{M}(\Lambda)$ могут состоять из конечного числа множеств $T_{j}$, которые всегда можно дополнить пустьми множествами до счетной последовательности).

Следуюшая лемма является следствием леммы 3 из [8].

Лемма 1. Пусть $\left\{T_{j}\right\} \in \mathfrak{M}(\mathbb{R}) \quad u f_{j} \in S(\mathbb{R}, \mathscr{U}), j \in \mathbb{N}$. Тогда

$$
\sum f_{j}(\cdot) \chi_{T_{j}}(\cdot) \in S(\mathbb{R}, \mathscr{U})
$$

$u$

$$
\operatorname{Mod}\left(\sum f_{j}(\cdot) \chi_{T_{j}}(\cdot)\right) \subset \sum_{j} \operatorname{Mod} f_{j}+\sum_{j} \operatorname{Mod} T_{j} .
$$

Множество непрерывных функций $\mathscr{F}: \mathscr{U} \rightarrow \mathbb{R}$ обозначим через $C(\mathscr{U}), C_{b}(\mathscr{U})$ - банахово пространство ограниченньх функций $\mathscr{F} \in C(\mathscr{U})$ с нормой

$$
\|\mathscr{F}\|=\|\mathscr{F}\|_{C_{b}(\mathscr{U})}=\sup _{x \in \mathscr{U}}|\mathscr{F}(x)|,
$$

$C_{b}^{+}(\mathscr{U})$ - множество неотрищательных функций из $C_{b}(\mathscr{U})$. Если $A \subset \mathscr{U}$, то $\mathscr{F}\left(\left.\cdot\right|_{A}\right)-$ ограничение функции $\mathscr{F} \in C(\mathscr{U})$ на множество $A$. По определению $S\left(\mathbb{R}, C_{b}(\mathscr{U})\right)=$ $S_{1}\left(\mathbb{R},\left(C_{b}(\mathscr{U}), \widetilde{D}\right)\right)$, где $\widetilde{D}\left(\mathscr{F}_{1}, \mathscr{F}_{2}\right)=\theta\left(\left\|\mathscr{F}_{1}-\mathscr{F}_{2}\right\|\right), \mathscr{F}_{1}, \mathscr{F}_{2} \in C_{b}(\mathscr{U})$.

Лемма 2. Пусть $(\mathscr{U}, \rho)$ - полное метрическое пространство. Если $f \in S(\mathbb{R}, \mathscr{U})$ и функиия $t \rightarrow \mathscr{F}(\cdot, t) \in C_{b}(\mathscr{U}), t \in \mathbb{R}$, принадлежст пространству $S\left(\mathbb{R}, C_{b}(\mathscr{U})\right)$, тогда

$$
\mathscr{F}(f(\cdot), \cdot) \in S \quad u \quad \operatorname{Mod} \mathscr{F}(f(\cdot), \cdot) \subset \operatorname{Mod} f+\operatorname{Mod} \mathscr{F}(\cdot, \cdot)
$$


ДОКАЗАТЕЛЬСТВО несложной леммы 2 можно найти в [8] и [15].

Лемма 3. Пусть $f \in S$. Тогда для любого $\varepsilon>0$ найдется множсество $T=$ $T(\varepsilon) \in S(\mathbb{R})$ maкое, ито $\operatorname{Mod} T \subset \operatorname{Mod} f, f(t)<2 \varepsilon$ nри всех $t \in T u f(t)>\varepsilon$ nри n.в. $t \in \mathbb{R} \backslash T$.

Лемма 3 непосредственно вытекает из теоремы 2 в [16].

Пусть $\mathscr{B}(\mathscr{U})$ - совокупность борелевских подмножеств метрического пространства $(\mathscr{U}, \rho), \mathscr{M}=\mathscr{M}(\mathscr{U})$ - множество борелевских вероятностных мер на пространстве $(\mathscr{U}, \rho)$. Каждой мере $\mu[\cdot] \in \mathscr{M}$ ставится в соответствие линейньй вероятностный функционал $\mu(\cdot)$ на банаховом пространстве

$$
C_{b}(\mathscr{U}): \mu(\mathscr{F})=\int_{\mathscr{U}} \mathscr{F}(x) \mu[d x], \quad \mathscr{F} \in C_{b}(\mathscr{U})
$$

Так как пространство $(\mathscr{U}, \rho)$ сепарабельно, то каждая мера $\mu[\cdot] \in \mathscr{M}$ является радоновой, т.е. для любого $\varepsilon>0$ существует компакт $K_{\varepsilon} \subset \mathscr{U}$ такой, что $\mu\left[K_{\varepsilon}\right]>1-\varepsilon$.

На множестве $\mathscr{M}$ вводится метрика Прохорова

$$
\widetilde{d}(\mu, \gamma)=\inf \left\{\varepsilon>0: \mu[A] \leqslant \gamma\left[A^{\varepsilon}\right]+\varepsilon \text { для всех } A \in \mathscr{B}(\mathscr{U})\right\}, \quad \mu, \gamma \in \mathscr{M},
$$

где $A^{\varepsilon}=\{x \in \mathscr{U}: \rho(x, A)<\varepsilon\}$. Метрическое пространство $(\mathscr{M}, \widetilde{d})$ является полньм и сепарабельным (вместе с пространством $(\mathscr{U}, \rho))$. Пусть $\mu_{j} \in \mathscr{M}, j \in \mathbb{N}$. Если $\mu \in \mathscr{M}$ и $\widetilde{d}\left(\mu, \mu_{j}\right) \rightarrow 0$ при $j \rightarrow+\infty$, то $\mu_{j}(\mathscr{F}) \rightarrow \mu(\mathscr{F})$ для любой функции $\mathscr{F} \in C_{b}(\mathscr{U})$. Наоборот, если $\varphi(\cdot)$ - линейньй вероятностньй функционал на $C_{b}(\mathscr{U})$ и $\mu_{j}(\mathscr{F}) \rightarrow \varphi(\mathscr{F})$ при $j \rightarrow+\infty$ для любой функции $\mathscr{F} \in C_{b}(\mathscr{U})$, то найдется мера $\mu \in \mathscr{M}$ такая, что $\varphi(\cdot)=\mu(\cdot)$ и $\widetilde{d}\left(\mu, \mu_{j}\right) \rightarrow 0$ при $j \rightarrow+\infty$.

Множество $\mathscr{K}$ предкомпактно в метрическом пространстве $(\mathscr{M}, \widetilde{d})$ тогда и только тогда, когда для любого $\varepsilon>0$ существует компакт $K_{\varepsilon} \subset \mathscr{U}$ такой, что $\mu\left[K_{\varepsilon}\right]>1-\varepsilon$ для всех мер $\mu \in \mathscr{K}$.

Обозначим через $\mathscr{L}(\mathscr{U})$ множество функций $\mathscr{F} \in C_{b}(\mathscr{U})$ таких, что $0 \leqslant \mathscr{F}(x) \leqslant 1$ для всех $x \in \mathscr{U}$ и $|\mathscr{F}(x)-\mathscr{F}(y)| \leqslant \rho(x, y)$ для всех $x, y \in \mathscr{U}$. Положим

$$
d(\mu, \gamma)=\sup \{|\mu(\mathscr{F})-\gamma(\mathscr{F})|: \mathscr{F} \in \mathscr{L}(\mathscr{U})\}, \quad \mu, \gamma \in \mathscr{M} .
$$

Метрики $d$ и $\widetilde{d}$ определяют одну и ту же топологию на $\mathscr{M}$. Более того, нетрудно показать, что для всех мер $\mu, \gamma \in \mathscr{M}$ выполняются неравенства

$$
\widetilde{d}^{2}(\mu, \gamma) \leqslant d(\mu, \gamma) \leqslant 24 \widetilde{d}^{1 / 3}(\mu, \gamma)
$$

поэтому приведенные для метрического пространства $(\mathscr{M}, \widetilde{d})$ свойства относятся также и к метрическому пространству $(\mathscr{M}, d)$. В настоящей работе будет использоваться (как более удобная) метрика $d$.

2. Мерозначная функция $t \rightarrow \mu[\cdot ; t] \in \mathscr{M}, t \in \mathbb{R}$, измерима, если $\mu[\cdot ; \cdot] \in M(\mathbb{R},(\mathscr{M}, d))$. 
ОПрЕДЕЛЕниЕ 1. Мерозначная функция $\mu[\cdot ; \cdot] \in M(\mathbb{R},(\mathscr{M}, d))$ называется почти периодической по Степанову, если $\mu[\cdot ; \cdot] \in S(\mathbb{R},(\mathscr{M}, d))($ так как $d(\mu, \gamma) \leqslant 1$ для всех мер $\mu, \gamma \in \mathscr{M}$, то $\theta(d(\cdot, \cdot))=d(\cdot, \cdot)$ и $\left.S(\mathbb{R},(\mathscr{M}, d))=S_{1}(\mathbb{R},(\mathscr{M}, d))\right)$.

Из неравенств $(1)$ следует, что $S(\mathbb{R},(\mathscr{M}, d))=S(\mathbb{R},(\mathscr{M}, \widetilde{d}))$ и $\operatorname{Mod} \mu[\cdot ; \cdot]$ для п.п. по Степанову мерозначной функции $\mu[\cdot ; \cdot]$ не зависит от того, какая из двух метрик на $\mathscr{M}$ рассматривается. В дальнейшем будет использоваться краткое обозначение $(\mathscr{M}, d)=\mathscr{M}$.

В [4], [9] определялись п.п. по Степанову мерозначные функции $t \rightarrow \mu[\cdot ; t] \in \mathscr{M}$, $t \in \mathbb{R}$, в случае, когда $\mathscr{U}$ - компакт в конечномерном евклидовом пространстве, при этом на множестве вероятностньг мер $\mathscr{M}(\mathscr{U})$ вводилась слабая метрика $d_{w}[1$, c. 53$]$. Так как в последнем случае компактные метрические пространства $\left(\mathscr{M}, d_{w}\right)$ и $(\mathscr{M}, d)$ гомеоморфны (при тождественном отображении множества $\mathscr{M}$ на себя), то эти функции совпадают с функциями из определения 1).

ЛЕмма 4. Если $\mu[\cdot ; \cdot] \in S(\mathbb{R}, \mathscr{M})$ и функиия $t \rightarrow \mathscr{F}(\cdot, t) \in C_{b}(\mathscr{U}), t \in \mathbb{R}$, принадлежит пространству $S\left(\mathbb{R}, C_{b}(\mathscr{U})\right)$, то функиия $t \rightarrow \mu(\mathscr{F}(\cdot, t) ; t), t \in \mathbb{R}$, принадлежит пространству $S$ и $\operatorname{Mod} \mu(\mathscr{F}(\cdot, \cdot) ; \cdot) \subset \operatorname{Mod} \mu[\cdot ; \cdot]+\operatorname{Mod} \mathscr{F}(\cdot, \cdot)$.

ДокАЗАТЕЛЬСТво. Каждой функции $\mathscr{F} \in C_{b}(\mathscr{U})$ соответствует функция $l_{\mathscr{F}}(\cdot) \in$ $C_{b}(\mathscr{M})$, для которой $l_{\mathscr{F}}(\mu)=\mu(\mathscr{F}), \mu \in \mathscr{M}$. При этом $\left\|l_{\mathscr{F}}\right\|_{C_{b}(\mathscr{M})}=\|\mathscr{F}\|_{C_{b}(\mathscr{U})}$ для всех функций $\mathscr{F} \in C_{b}(\mathscr{U})$. Из последнего равенства следует, что функция $t \rightarrow l_{\mathscr{F}(\cdot, t)}(\cdot)$, $t \in \mathbb{R}$, принадлежит пространству $S\left(\mathbb{R}, C_{b}(\mathscr{M})\right)$ и $\operatorname{Mod} l_{\mathscr{F}(\cdot, \cdot)}(\cdot)=\operatorname{Mod} \mathscr{F}(\cdot, \cdot)$. Так как метрическое пространство $\mathscr{M}$ полное и $\mu[\cdot ; \cdot] \in S(\mathbb{R}, \mathscr{M})$, то отсюда в силу леммы 2 получаем $l_{\mathscr{F}(\cdot, \cdot)}(\mu[\cdot ; \cdot])=\mu(\mathscr{F}(\cdot, \cdot) ; \cdot) \in S$ и

$$
\operatorname{Mod} \mu(\mathscr{F}(\cdot, \cdot) ; \cdot) \subset \operatorname{Mod} \mu[\cdot ; \cdot]+\operatorname{Mod} l_{\mathscr{F}(\cdot, \cdot)}(\cdot)=\operatorname{Mod} \mu[\cdot ; \cdot]+\operatorname{Mod} \mathscr{F}(\cdot, \cdot) .
$$

Лемма доказана.

СледСТВИЕ. $Е с л и \mu[\cdot ; \cdot] \in S(\mathbb{R}, \mathscr{M})$, то для любой функиии $\mathscr{F} \in C_{b}(\mathscr{U})$ имеем $\mu(\mathscr{F} ; \cdot) \in S_{1} u \operatorname{Mod} \mu(\mathscr{F} ; \cdot) \subset \operatorname{Mod} \mu[\cdot ; \cdot]$.

Теорема 1. Пусть $(\mathscr{U}, \rho)$ - полное сепарабельное метрическое пространство $u t \rightarrow \mu[\cdot ; t] \in \mathscr{M}, t \in \mathbb{R},-$ произвольная функиия. Предположим, что $\mu(\mathscr{F} ; \cdot) \in S$ для всех функиий $\mathscr{F} \in C_{b}(\mathscr{U})$. Тогда $\mu[\cdot ; \cdot] \in S(\mathbb{R}, \mathscr{M}) u$

$$
\operatorname{Mod} \mu[\cdot ; \cdot]=\sum_{\mathscr{F} \in C_{b}(\mathscr{U})} \operatorname{Mod} \mu(\mathscr{F} ; \cdot) \text {. }
$$

Доказательство теоремы 1 приведено в п. 3.

В теореме 1 , в частности, содержится утверждение о том, что произвольная мерозначная функция $t \rightarrow \mu[\cdot ; t] \in \mathscr{M}, t \in \mathbb{R}$, измерима, если для всех $\mathscr{F} \in C_{b}(\mathscr{U})$ измеримы функции $\mu(\mathscr{F} ; \cdot)$.

Лемма 5. Пусть $\mu[\cdot ; \cdot] \in S(\mathbb{R}, \mathscr{M})$. Тогда существует функиия $\mathscr{F} \in C_{b}(\mathscr{U})$ такая, что $\operatorname{Mod} \mu[\cdot ; \cdot]=\operatorname{Mod} \mu(\mathscr{F} ; \cdot)$.

ДоказАтЕЛЬСтво. Будем считать, что $\operatorname{Mod} \mu[\cdot ; \cdot] \neq\{0\}$. Пусть $\lambda_{j}, j \in \mathbb{N},-$ как-то перенумерованные числа из счетного множества $\operatorname{Mod} \mu[\cdot ; \cdot] \backslash\{0\}$. Каждому индексу $j \in \mathbb{N}$ поставим в соответствие какую-либо функцию $\mathscr{F}_{j} \in C_{b}^{+}(\mathscr{U})$, для которой $\lambda_{j} \in \operatorname{Mod} \mu\left(\mathscr{F}_{j} ; \cdot\right)$ (это можно сделать в силу теоремы 1$)$. Положим $\alpha_{1}=1$. Найдется число $\beta_{1}>0$ такое, что $\lambda_{1} \in \operatorname{Mod} \mu\left(\alpha_{1} \mathscr{F}_{1}+\widetilde{\mathscr{F}} ; \cdot\right)$ для всех функций $\widetilde{\mathscr{F}} \in C_{b}(\mathscr{U})$, для 
которых $\|\widetilde{\mathscr{F}}\| \leqslant \beta_{1}$. Далее последовательно при $j=2,3, \ldots$ будем определять числа $\alpha_{j}>0$ и $\beta_{j}>0$. Если при некотором $j \in \mathbb{N}$ числа $\alpha_{1}, \ldots, \alpha_{j}$ и $\beta_{1}, \ldots, \beta_{j}$ уже определены, причем

$$
\lambda_{j} \in \operatorname{Mod} \mu\left(\sum_{k=1}^{j} \alpha_{k} \mathscr{F}_{k}+\widetilde{\mathscr{F}} ; \cdot\right)
$$

для всех функций $\widetilde{\mathscr{F}} \in C_{b}(\mathscr{U})$, для которых $\|\widetilde{\mathscr{F}}\| \leqslant \beta_{j}$, то выберем числа $\alpha_{j+1}$ и $\beta_{j+1}$ так, что $\alpha_{j+1}\left\|\mathscr{F}_{j+1}\right\|+\beta_{j+1} \leqslant \beta_{j}$ и $\lambda_{j+1} \in \operatorname{Mod} \mu\left(\sum_{k=1}^{j+1} \alpha_{k} \mathscr{F}_{k}+\widetilde{\mathscr{F}} ; \cdot\right)$ для всех функций $\widetilde{\mathscr{F}} \in C_{b}(\mathscr{U})$, для которых $\|\widetilde{\mathscr{F}}\| \leqslant \beta_{j+1}$ (это можно сделать, так как $\operatorname{Mod} f$ для любой функции $f \in S_{1}$ совпадает с модулем ее показателей Фурье, которые устойчивы при малых возмущениях функции $f$ ). Продолжим до бесконечности нахождение чисел $\alpha_{j}$ и $\beta_{j}$. Обозначим $\mathscr{F}=\sum_{k} \alpha_{k} \mathscr{F}_{k}$. Из условий, наложенных на числа $\alpha_{j}$ и $\beta_{j}$, следует, что $\mathscr{F} \in C_{b}(\mathscr{U})$ и $\lambda_{j} \in \operatorname{Mod} \mu(\mathscr{F} ; \cdot)$ для всех $j \in \mathbb{N}$. Учитывая следствие леммы 4 , отсюда получаем, что $\operatorname{Mod} \mu[\cdot ; \cdot]=\operatorname{Mod} \mu(\mathscr{F} ; \cdot)$. Лемма доказана.

3. Для доказательства теоремы 1 потребуется ряд вспомогательных утверждений.

Пусть $B$ - банахово пространство п.п. по Бору функций $f: \mathbb{R} \rightarrow \mathbb{R}$ (см. [11], [12]) с нормой $\|f\|_{B}=\sup _{t \in \mathbb{R}}|f(t)|$. Обозначим

$$
\|f\|_{S_{1}}=\sup _{\xi \in \mathbb{R}} \int_{\xi}^{\xi+1}|f(t)| d t,
$$

если $f \in S_{1}$.

Теорема 2. Пусть $f_{j} \in B, j \in \mathbb{N}, f_{j}(t) \geqslant 0$ для всех $t \in \mathbb{R}$. Предположим, что $\sum_{j \in J} f_{j}(\cdot) \in B$ для любого множества $J \subset \mathbb{N}$. Обозначим $g_{j}(\cdot)=\sum_{i \geqslant j} f_{i}(\cdot)$, $j \in \mathbb{N}$. Тогда $\left\|g_{j}\right\|_{B} \rightarrow 0$ при $j \rightarrow+\infty$.

ДоказАтЕльство. Допустим противное. Так как $0 \leqslant \cdots \leqslant g_{j+1}(t) \leqslant g_{j}(t) \leqslant \cdots$ $\leqslant g_{1}(t)$ для всех $t \in \mathbb{R}$, то последовательность $\left\|g_{j}\right\|_{B}, j \in \mathbb{N}$, не возрастает и, следовательно, существует число $\varepsilon>0$ такое, что $\left\|g_{j}\right\|_{B} \geqslant \varepsilon$ при всех $j \in \mathbb{N}$. Положим $j_{1}=1$. Найдется число $t_{1} \in \mathbb{R}$ такое, что $g_{j_{1}}\left(t_{1}\right) \geqslant \frac{3}{4} \varepsilon$. Выберем индекс $j_{1}^{\prime} \in \mathbb{N}, j_{1}^{\prime}>j_{1}$, так, чтобы вьполнялось неравенство $g_{j_{1}}\left(t_{1}\right)-g_{j_{1}^{\prime}}\left(t_{1}\right) \geqslant \varepsilon / 2$. Обозначим $G_{1}(\cdot)=g_{j_{1}}(\cdot)-g_{j_{1}^{\prime}}(\cdot)$. После этого выберем число $\alpha_{1} \geqslant 1$ так, чтобы для любого $\xi \in \mathbb{R}$ нашелся 1-почти период $\tau=\tau(\xi)$ функции $G_{1}(\cdot)$, для которого $\xi-\tau \in\left[t_{1}, t_{1}+\alpha_{1}\right]$. Будем, далее, последовательно (при $s=2,3, \ldots$ ) находить числа $t_{s} \in \mathbb{R}$, индексы $j_{s}, j_{s}^{\prime}>j_{s}$ и числа $\alpha_{s} \geqslant s$. Предположим, что они уже определены при некотором $s \in \mathbb{N}$, при этом $G_{n}(\cdot)=g_{j_{n}}(\cdot)-g_{j_{n}^{\prime}}(\cdot), n=1, \ldots, s$. Из условий теоремы и леммы Дини следует, что функции $g_{j}(\cdot)$ стремятся к 0 при $j \rightarrow+\infty$ равномерно на каждом отрезке, поэтому можно выбрать индекс $j_{s+1} \geqslant j_{s}^{\prime}$ так, чтобы для всех $t \in\left[t_{s}-\alpha_{s}, t_{s}+\alpha_{s}\right]$ выполнялось неравенство $g_{j_{s+1}}(t) \leqslant 1 / s$. Найдется число $t_{s+1} \in \mathbb{R}$, для которого $g_{j_{s+1}}\left(t_{s+1}\right) \geqslant \frac{3}{4} \varepsilon$. Выберем индекс $j_{s+1}^{\prime}>j_{s+1}$ так, что

$$
g_{j_{s+1}}\left(t_{s+1}\right)-g_{j_{s+1}^{\prime}}\left(t_{s+1}\right) \geqslant \frac{\varepsilon}{2} .
$$

Обозначим

$$
G_{s+1}(\cdot)=g_{j_{s+1}}(\cdot)-g_{j_{s+1}^{\prime}}(\cdot) .
$$

После этого выберем число $\alpha_{s+1}$ из следующих условий:

1) $\alpha_{s+1} \geqslant s+1$; 
2) $\left[t_{s}, t_{s}+\alpha_{s}\right] \subset\left[t_{s+1}-\alpha_{s+1}, t_{s+1}+\alpha_{s+1}\right]$;

$3)$ для любого $\xi \in \mathbb{R}$ найдется $1 /(s+1)$-почти период $\tau=\tau(\xi)$ функции

$$
\sum_{n \leqslant s+1} G_{n}(\cdot) \in B
$$

для которого $\xi-\tau \in\left[t_{s+1}, t_{s+1}+\alpha_{s+1}\right]$.

Продолжим неограниченно нахождение чисел $t_{s} \in \mathbb{R}$, индексов $j_{s}, j_{s}^{\prime}$ и чисел $\alpha_{s} \geqslant s$.

Обозначим

$$
\mathscr{H}_{s}(\cdot)=\sum_{n \geqslant s} G_{n}(\cdot)=\sum_{n \geqslant s}\left(f_{j_{n}}(\cdot)+\cdots+f_{j_{n}^{\prime}-1}(\cdot)\right), \quad s \in \mathbb{N} .
$$

Имеем $\mathscr{H}_{s} \in B, \mathscr{H}_{s}(t) \leqslant g_{j_{s}}(t)$ при всех $t \in \mathbb{R}$ и $\mathscr{H}_{s}\left(t_{s}\right) \geqslant G_{s}\left(t_{s}\right) \geqslant \varepsilon / 2$ при всех $s \in \mathbb{N}$. В дальнейшем будет также использоваться обозначение

$$
\mathscr{P}_{s}(\cdot)=\sum_{n \leqslant s} G_{n}(\cdot)=\mathscr{H}_{1}(\cdot)-\mathscr{H}_{s+1}(\cdot), \quad s \in \mathbb{N} .
$$

Пусть $L$ - такое положительное число, что на каждом отрезке длины $L$ имеется по крайней мере один $\varepsilon / 32$-почти период функции $\mathscr{H}_{1}(\cdot)$. Выберем число $N \in \mathbb{N}$, исходя из условий $2 N \geqslant L$ и $\varepsilon N \geqslant 8$. Для всех $t \in\left[t_{N}-\alpha_{N}, t_{N}+\alpha_{N}\right]$ имеем

$$
\mathscr{H}_{N+1}(t) \leqslant g_{j_{N+1}}(t) \leqslant \frac{1}{N} .
$$

Пусть $L^{\prime}>0$ - такое число, что на любом отрезке длины $L^{\prime}$ существует по крайней мере один $1 / N$-почти период функции $\mathscr{H}_{N+1}(\cdot)$. Тогда для любого $s \in \mathbb{N}$ можно найти число $\tau_{s}^{\prime} \in\left[-L^{\prime} / 2, L^{\prime} / 2\right]$ такое, что $t_{s}+\tau_{s}^{\prime}-t_{N}$ есть $1 / N$-почти период функции $\mathscr{H}_{N+1}(\cdot)$. Отсюда для всех $s \geqslant N+1$ и $t \in\left[t_{s}+\tau_{s}^{\prime}-\alpha_{N}, t_{s}+\tau_{s}^{\prime}+\alpha_{N}\right]$ получаем

$$
\begin{aligned}
\mathscr{H}_{s}(t) & \leqslant \mathscr{H}_{N+1}(t)=\left(\mathscr{H}_{N+1}(t)-\mathscr{H}_{N+1}\left(t-t_{s}-\tau_{s}^{\prime}+t_{N}\right)\right)+\mathscr{H}_{N+1}\left(t-t_{s}-\tau_{s}^{\prime}+t_{N}\right) \\
& <\frac{1}{N}+\frac{1}{N}=\frac{2}{N} .
\end{aligned}
$$

С другой стороны, так как $2 \alpha_{N} \geqslant 2 N \geqslant L$, то для каждого $s \geqslant N+1$ можно найти $\varepsilon / 32$-почти период $\tau_{s}$ функции $\mathscr{H}_{1}(\cdot)$, для которого $t_{s}+\tau_{s} \in\left[t_{s}+\tau_{s}^{\prime}-\alpha_{N}, t_{s}+\tau_{s}^{\prime}+\alpha_{N}\right]$. Следовательно, для всех $s \geqslant N+1$ имеем

$$
\left\|\mathscr{H}_{s}\left(\cdot+\tau_{s}\right)-\mathscr{H}_{s}(\cdot)\right\|_{B} \geqslant \mathscr{H}_{s}\left(t_{s}\right)-\mathscr{H}_{s}\left(t_{s}+\tau_{s}\right)>\frac{\varepsilon}{2}-\frac{2}{N} \geqslant \frac{\varepsilon}{4}
$$

и $\left|\tau_{s}\right| \leqslant\left|\tau_{s}^{\prime}\right|+\alpha_{N} \leqslant L^{\prime} / 2+\alpha_{N}$.

Выберем теперь число $M \in \mathbb{N}$, исходя из условий $M \geqslant L^{\prime} / 2+\alpha_{N}$ и $\varepsilon M \geqslant 64$ (тогда $\left|\tau_{s}\right| \leqslant M$ при всех $\left.s \geqslant N+1\right)$. Пусть $s \geqslant \max \{N, M\}$. Фиксируем некоторое число $\eta \in \mathbb{R}$. Пусть $\widetilde{\tau}$ - такой $1 / s$-почти период функции $\mathscr{P}_{s}(\cdot)$, что

$$
\eta+\left|\tau_{s+1}\right|-\tilde{\tau} \in\left[t_{s}, t_{s}+\alpha_{s}\right] .
$$

Так как $\alpha_{s} \geqslant s \geqslant M \geqslant\left|\tau_{s+1}\right|$, то

$$
\eta-\widetilde{\tau} \in\left[t_{s}-\alpha_{s}, t_{s}+\alpha_{s}\right] .
$$


Имеем

$$
\begin{gathered}
\left|\mathscr{P}_{s}\left(\eta+\left|\tau_{s+1}\right|\right)-\mathscr{P}_{s}\left(\eta+\left|\tau_{s+1}\right|-\tilde{\tau}\right)\right|<\frac{1}{s} \\
\left|\mathscr{P}_{s}(\eta)-\mathscr{P}_{s}(\eta-\widetilde{\tau})\right|<\frac{1}{s}
\end{gathered}
$$

Справедливо равенство

$$
\begin{aligned}
\mathscr{H}_{s+1} & \left(\eta+\left|\tau_{s+1}\right|\right)-\mathscr{H}_{s+1}(\eta) \\
= & \mathscr{H}_{1}\left(\eta+\left|\tau_{s+1}\right|\right)-\mathscr{H}_{1}(\eta)-\left(\mathscr{P}_{s}\left(\eta+\left|\tau_{s+1}\right|\right)-\mathscr{P}_{s}\left(\eta+\left|\tau_{s+1}\right|-\widetilde{\tau}\right)\right) \\
& +\left(\mathscr{P}_{s}(\eta)-\mathscr{P}_{s}(\eta-\widetilde{\tau})\right)-\left(\mathscr{P}_{s}\left(\eta+\left|\tau_{s+1}\right|-\widetilde{\tau}\right)-\mathscr{P}_{s}(\eta-\widetilde{\tau})\right) .
\end{aligned}
$$

Так как $\mathscr{H}_{s+1}(t) \leqslant g_{j_{s+1}}(t) \leqslant 1 / s$ для всех $t \in\left[t_{s}-\alpha_{s}, t_{s}+\alpha_{s}\right]$, то отсюда и из неравенств (5), (6), учитьвая (3), (4), получаем

$$
\begin{aligned}
\left|\mathscr{H}_{s+1}\left(\eta+\left|\tau_{s+1}\right|\right)-\mathscr{H}_{s+1}(\eta)\right| & <\frac{\varepsilon}{32}+\frac{2}{s}+\left|\mathscr{P}_{s}\left(\eta+\left|\tau_{s+1}\right|-\widetilde{\tau}\right)-\mathscr{P}_{s}(\eta-\widetilde{\tau})\right| \\
\leqslant & \frac{\varepsilon}{32}+\frac{2}{s}+\left|\mathscr{H}_{1}\left(\eta+\left|\tau_{s+1}\right|-\widetilde{\tau}\right)-\mathscr{H}_{1}(\eta-\widetilde{\tau})\right| \\
& +\mathscr{H}_{s+1}\left(\eta+\left|\tau_{s+1}\right|-\widetilde{\tau}\right)+\mathscr{H}_{s+1}(\eta-\widetilde{\tau}) \\
& <\frac{\varepsilon}{16}+\frac{4}{M} \leqslant \frac{\varepsilon}{8} .
\end{aligned}
$$

Число $\eta \in \mathbb{R}$ можно выбрать произвольно, поэтому из полученных неравенств при всех $s \geqslant \max \{N, M\}$ следует оценка

$$
\left\|\mathscr{H}_{s+1}\left(\cdot+\tau_{s+1}\right)-\mathscr{H}_{s+1}(\cdot)\right\|_{B} \leqslant \frac{\varepsilon}{8} .
$$

Но последняя оценка противоречит неравенству (2), которое справедливо при всех $s \geqslant N+1$. Поэтому сделанное предположение о том, что $\left\|g_{j}\right\|_{B} \geqslant \varepsilon>0$ при всех $j \in \mathbb{N}$, неверно. Теорема 2 доказана.

СледствиЕ. Пусть $f_{j} \in S_{1}, j \in \mathbb{N}, u f_{j}(t) \geqslant 0$ при п.в. $t \in \mathbb{R}$. Предположим, что $\sum_{j \in J} f_{j}(\cdot) \in S_{1}$ для любого множсества $J \subset \mathbb{N}$. Обозначим $g_{j}(\cdot)=\sum_{i \geqslant j} f_{i}(\cdot)$, $j \in \mathbb{N}$. Tогда $\left\|g_{j}\right\|_{S_{1}} \rightarrow 0$ при $j \rightarrow+\infty$.

Для доказательства следствия достаточно рассмотреть функции

$$
\tilde{f}_{j}(\cdot)=\int_{-1 / 2}^{1 / 2} f_{j}(\cdot+t) d t \in B, \quad j \in \mathbb{N} .
$$

Если $\mathscr{F} \in C_{b}(\mathscr{U})$ и функция $\mu[\cdot ; \cdot]$ удовлетворяет условиям теоремы 1 , то в силу ограниченности функции $\mu(\mathscr{F} ; \cdot) \in S$ имеем $\mu(\mathscr{F} ; \cdot) \in S_{1}$.

ТЕОремА 3. Пусть мерозначная функиия $\mu[\cdot ; \cdot]$ удовлетворяет условиям теоремы $1, \mathscr{F}_{j} \in C_{b}^{+}(\mathscr{U}), j \in \mathbb{N}, 0 \leqslant \cdots \leqslant \mathscr{F}_{j+1}(x) \leqslant \mathscr{F}_{j}(x) \leqslant \cdots \leqslant \mathscr{F}_{1}(x) u \mathscr{F}_{j}(x) \rightarrow 0$ при $j \rightarrow+\infty$ для всех $x \in \mathscr{U}$. Тогда $\|\mu(\mathscr{F} ; \cdot)\|_{S_{1}} \rightarrow 0$ при $j \rightarrow+\infty$. 
ДоказАТЕЛЬСтво. Обозначим $h_{j}(\cdot)=\mathscr{F}_{j}(\cdot)-\mathscr{F}_{j+1}(\cdot), j \in \mathbb{N}$. Тогда $\mu\left(h_{j} ; \cdot\right) \in S_{1}$ и $\mu\left(h_{j} ; t\right) \geqslant 0$ при п.в. $t \in \mathbb{R}$. Для произвольного множества $J \subset \mathbb{N}$ положим

$$
h(J ; \cdot)=\sum_{j \in J} h_{j}(\cdot) .
$$

Нетрудно убедиться, что $h(J ; \cdot) \in C_{b}^{+}(\mathscr{U})$, поэтому $\mu(h(J ; \cdot) ; \cdot) \in S_{1}$. С другой стороны,

$$
\sum_{j \in J, j \leqslant s} h_{j}(x) \rightarrow h(J ; x)
$$

при $s \rightarrow+\infty$ для всех $x \in \mathscr{U}$, поэтому

$$
\mu(h(J ; \cdot) ; t)=\sum_{j \in J} \mu\left(h_{j} ; t\right)
$$

п.в. и, следовательно,

$$
\sum_{j \in J} \mu\left(h_{j} ; \cdot\right) \in S_{1}
$$

Осталось воспользоваться следствием теоремы 2. Теорема 3 доказана.

Для функции $\mu[\cdot ; \cdot]$, удовлетворяющей условиям теоремы 1 , обозначим

$$
\Lambda(\mu)=\sum_{\mathscr{F} \in C_{b}(\mathscr{U})} \operatorname{Mod} \mu(\mathscr{F} ; \cdot) .
$$

Лемма 6. Пусть мерозначная функиия $\mu[\cdot ; \cdot]$ удовлетворяет условиям теоремы 1. Тогда для любого $\varepsilon>0$ существует множество $T=T(\varepsilon) \in S(\mathbb{R})$ такое, что $\operatorname{Mod} T \subset \Lambda(\mu), \varkappa(T)<\varepsilon u \mathscr{K}=\bigcup_{t \in T} \mu[\cdot ; t]-$ компакт в метрическом пространстве $(\mathscr{M}, d)$.

ДокАЗАТЕЛЬСтво. Пусть $\left\{x_{i}\right\}_{i \in \mathbb{N}}$ - плотное в пространстве $\mathscr{U}$ множество точек. Для всех $j, n \in \mathbb{N}$ определим функции

$$
\mathscr{F}_{j}^{(n)}(x)=\theta\left(2 n \rho\left(x, \bigcup_{i \leqslant j} x_{i}\right)-1\right), \quad x \in \mathscr{U} .
$$

Так как $\mathscr{F}_{j}^{(n)}(\cdot) \in C_{b}^{+}(\mathscr{U})$, то $\mu\left(\mathscr{F}_{j}^{(n)} ; \cdot\right) \in S_{1}$. Функции $\mathscr{F}_{j}^{(n)}$ (при фиксированном $n \in \mathbb{N}$ ) удовлетворяют условиям теоремы 3 , поэтому для каждого $n \in \mathbb{N}$ существует номер $j(n) \in \mathbb{N}$ такой, что $\left\|\mu\left(\mathscr{F}_{n} ; \cdot\right)\right\|_{S_{1}}<2^{-2 n} \varepsilon^{2}$, где $\mathscr{F}_{n}(\cdot)=\mathscr{F}_{j(n)}^{(n)}(\cdot)$. Из леммы 3 вытекает существование множества $T^{(n)} \in S(\mathbb{R})$, для которого $\operatorname{Mod} T^{(n)} \subset \operatorname{Mod} \mu\left(\mathscr{F}_{n} ; \cdot\right)$, $\mu\left(\mathscr{F}_{n} ; t\right)<2^{1-n} \varepsilon$ при всех $t \in T^{(n)}$ и $\mu\left(\mathscr{F}_{n} ; t\right)>2^{-n} \varepsilon$ при п.в. $t \in \mathbb{R} \backslash T^{(n)}$. Поэтому для любого $\xi \in \mathbb{R}$ имеем

$$
2^{-n} \varepsilon \operatorname{mes}\left([\xi, \xi+1] \backslash T^{(n)}\right) \leqslant\left\|\mu\left(\mathscr{F}_{n} ; \cdot\right)\right\|_{S_{1}}<2^{-2 n} \varepsilon^{2} .
$$

Отсюда $\varkappa\left(T^{(n)}\right)<2^{-n} \varepsilon$. Для каждого $m \in \mathbb{N}$ обозначим

$$
T_{m}=\bigcap_{n \geqslant m+1} T^{(n)} .
$$


Тогда $T_{m} \in S(\mathbb{R}), \operatorname{Mod} T_{m} \subset \sum_{n} \operatorname{Mod} T^{(n)} \subset \Lambda(\mu)$ и

$$
\varkappa\left(T_{m}\right) \leqslant \sum_{n \geqslant m+1} \varkappa\left(T^{(n)}\right)<2^{-m} \varepsilon .
$$

Пусть

$$
K_{m}=\bigcap_{n \geqslant m+1} \bigcup_{i \leqslant j(n)} K\left(x_{i}, 1 / n\right), \quad m \in \mathbb{N} .
$$

Для любого $m \in \mathbb{N}$ точки $x_{i}, i=1, \ldots, j(n)$, при $n \geqslant m+1$ образуют конечную $2 / n$-сеть для замкнутого множества $K_{m} \subset \mathcal{U}$, поэтому $K_{m}$ - компакт. Если $t \in T_{m}$, то при всех $n \geqslant m+1$ справедлива оценка

$$
\mu\left[\bigcup_{i \leqslant j(n)} K\left(x_{i}, \frac{1}{n}\right) ; t\right] \geqslant 1-\mu\left(\mathscr{F}_{n} ; t\right)>1-2^{1-n} \varepsilon,
$$

следовательно,

$$
\mu\left[K_{m} ; t\right] \geqslant 1-\sum_{n \geqslant m+1} \mu\left(\mathscr{F}_{n} ; t\right)>1-2^{1-m} \varepsilon .
$$

Положим $T=\bigcap_{m} T_{m}$. Имеем $T \in S(\mathbb{R})$,

$$
\operatorname{Mod} T \subset \sum_{m} \operatorname{Mod} T_{m} \subset \Lambda(\mu) \quad \text { и } \quad \varkappa(T) \leqslant \sum_{m} \varkappa\left(T_{m}\right)<\varepsilon .
$$

С другой стороны, для всех $m \in \mathbb{N}$ и $t \in T$ вьполняется неравенство $\mu\left[K_{m} ; t\right]>1-2^{1-m} \varepsilon$. Поэтому $\mathscr{K}=\overline{\bigcup_{t \in T} \mu[\cdot ; t]}$ - компакт в метрическом пространстве $(\mathscr{M}, d)$. Лемма доказана.

ДокаЗАТЕЛЬСТво тЕОРемы 1 . ВЫберем для всех $l \in \mathbb{N}$ (в соответствии с леммой 6) множества $T_{l}^{\prime} \in S(\mathbb{R})$ такие, что $\operatorname{Mod} T_{l}^{\prime} \subset \Lambda(\mu), \varkappa\left(T_{l}^{\prime}\right)<1 / l$ и $\bigcup_{t \in T_{l}^{\prime}} \mu[\cdot ; t]-$ предкомпактные множества в метрическом пространстве $(\mathscr{M}, d)$. Положим $T_{1}=T_{1}^{\prime}$, $T_{l}=T_{l}^{\prime} \backslash \bigcup_{s<l} T_{s}^{\prime}, l \geqslant 2$. Выбрасывая множества $T_{l}$, для которых $\operatorname{mes} T_{l}=0$, можно считать, что mes $T_{l} \neq 0$ для всех $l \in \mathbb{N}$ (возможно, останется только конечное число множеств $\left.T_{l}\right)$. Имеем $\left\{T_{l}\right\} \in \mathfrak{M}(\Lambda(\mu))$, при этом $\mathscr{K}_{l}=\overline{\bigcup_{t \in T_{l}} \mu[\cdot ; t]}$ - компактные множества в пространстве $(\mathscr{M}, d), l \in \mathbb{N}$.

Фиксируем число $l \in \mathbb{N}$. Пусть $K_{m}^{(l)} \subset \mathscr{U}$-компактныемножества, определенныепри доказательстве леммы 6 , такие, что $\mu\left[K_{m}^{(l)}\right] \geqslant 1-2^{1-m} / l$ для всех $\mu \in \mathscr{K}_{l}$ и $m \in \mathbb{N}$ (в условиях леммы 6 положено $\varepsilon=1 / l)$. Вместе с множествами $K_{m}^{(l)}$ компактньми являются также множества $\mathscr{L}\left(K_{m}^{(l)}\right)$ в банаховых пространствах $\left(C_{b}\left(K_{m}^{(l)}\right),\|\cdot\|_{C_{b}\left(K_{m}^{(l)}\right)}\right)$, поэтому (так как $\left.\mathscr{L}\left(K_{m}^{(l)}\right)=\left\{\mathscr{F}\left(\left.\cdot\right|_{K_{m}^{(l)}}\right): \mathscr{F} \in \mathscr{L}(\mathscr{U})\right\}\right)$ найдется последовательность функций $\mathscr{F}_{j} \in \mathscr{L}(\mathscr{U}), j \in \mathbb{N}$, такая, что множества $\left\{\mathscr{F}_{j}\left(\left.\cdot\right|_{K_{m}^{(l)}}\right)\right\}_{j \in \mathbb{N}}$ плотны в $\mathscr{L}\left(K_{m}^{(l)}\right) \subset$ $\left(C_{b}\left(K_{m}^{(l)}\right),\|\cdot\|_{C_{b}\left(K_{m}^{(l)}\right)}\right)$ для всех $m \in \mathbb{N}$. Из выбора множеств $K_{m}^{(l)}$ и функций $\mathscr{F}_{j}$ следует, что

$$
d^{0}(\mu, \gamma)=\sum_{j=1}^{+\infty} 2^{-j}\left|\mu\left(\mathscr{F}_{j}\right)-\gamma\left(\mathscr{F}_{j}\right)\right|,
$$


$\mu, \gamma \in \mathscr{K}_{l},-$ метрика на множестве $\mathscr{K}_{l}$. При этом $d^{0}(\mu, \gamma) \leqslant d(\mu, \gamma)$ для всех мер $\mu, \gamma \in \mathscr{K}_{l}$. Тождественное отображение компактного метрического пространства $\left(\mathscr{K}_{l}, d\right)$ на метрическое пространство $\left(\mathscr{K}_{l}, d^{0}\right)$ непрерывно, поэтому $\left(\mathscr{K}_{l}, d^{0}\right)$ - также компактное пространство и непрерывно обратное отображение [17, с. 190].

$\Phi$ иксируем произвольную точку $t_{l} \in T_{l}$. Положим

$$
\widetilde{\mu}_{l}[\cdot ; t]= \begin{cases}\mu[\cdot ; t], & \text { если } t \in T_{l}, \\ \mu\left[\cdot ; t_{l}\right], & \text { если } t \notin T_{l},\end{cases}
$$

$\widetilde{\mu}_{l}[\cdot ; t] \in \mathscr{K}_{l}$ при всех $t \in \mathbb{R}$. Из леммы 1 получаем $\widetilde{\mu}_{l}\left(\mathscr{F}_{j} ; \cdot\right) \in S$ и

$$
\operatorname{Mod} \widetilde{\mu}_{l}\left(\mathscr{F}_{j} ; \cdot\right) \subset \operatorname{Mod} \mu\left(\mathscr{F}_{j} ; \cdot\right)+\operatorname{Mod} T_{l} \subset \Lambda(\mu), \quad j \in \mathbb{N} .
$$

Из измеримости функций $\widetilde{\mu}_{l}\left(\mathscr{F}_{j} ; \cdot\right), j \in \mathbb{N}$, (и определения метрики $d^{0}$ ) вытекает измеримость функции $t \rightarrow d^{0}\left(\widetilde{\mu}_{l}[\cdot ; t], \gamma[\cdot]\right), t \in \mathbb{R}$, для любой меры $\gamma \in \mathscr{K}_{l}$. Так как пространство $\left(\mathscr{K}_{l}, d^{0}\right)$ сепарабельное, то отсюда следует измеримость функции $\widetilde{\mu}_{l}[\cdot ; \cdot]$ со значениями в метрическом пространстве $\left(\mathscr{K}_{l}, d^{0}\right)[14]$. Для любого $\delta>0$ выберем число $N(\delta) \in \mathbb{N}$ так, что $2^{-N(\delta)}<\delta / 2$. Если $\tau$ есть общий $\delta / 2$-почти период функций $\widetilde{\mu}_{l}\left(\mathscr{F}_{j} ; \cdot\right) \in S_{1}$ (множество таких чисел $\tau$ относительно плотно $\left.[11]\right), j=1, \ldots, N(\delta)$, то

$$
\begin{aligned}
D^{\left(d^{0}\right)}\left(\widetilde{\mu}_{l}[\cdot ; \cdot+\tau], \widetilde{\mu}_{l}[\cdot ; \cdot]\right) & \leqslant \sum_{j=1}^{N(\delta)} 2^{-j}\left\|\widetilde{\mu}_{l}\left(\mathscr{F}_{j} ; \cdot+\tau\right)-\tilde{\mu}_{l}\left(\mathscr{F}_{j} ; \cdot\right)\right\|_{S_{1}}+\sum_{j=N(\delta)+1}^{+\infty} 2^{-j} \\
& <\frac{\delta}{2}+\frac{\delta}{2}=\delta .
\end{aligned}
$$

Поэтому (в силу произвольности выбора числа $\delta>0) \widetilde{\mu}_{l}[\cdot ; \cdot] \in S\left(\mathbb{R},\left(\mathscr{K}_{l}, d^{0}\right)\right)$ и, более того, любая $\widetilde{\mu}_{l}\left(\mathscr{F}_{j} ; \cdot\right)$-возрастающая для всех $j \in \mathbb{N}$ последовательность $\left\{\tau_{j}\right\} \subset \mathbb{R}$ является также и $\widetilde{\mu}_{l}[\cdot ; \cdot \cdot]$-возрастающей. Следовательно,

$$
\operatorname{Mod} \widetilde{\mu}_{l}[\cdot ; \cdot] \subset \sum_{j=1}^{+\infty} \operatorname{Mod} \widetilde{\mu}_{l}\left(\mathscr{F}_{j} ; \cdot\right) \subset \Lambda(\mu) \text {. }
$$

В силу непрерьвности тождественного отображения компактного метрического пространства $\left(\mathscr{K}_{l}, d^{0}\right)$ на пространство $\left(\mathscr{K}_{l}, d\right)$ получаем $\widetilde{\mu}_{l}[\cdot ; \cdot] \in S(\mathbb{R},(\mathscr{M}(\mathscr{U}), d))$ $\left(\operatorname{Mod} \widetilde{\mu}_{l}[\cdot ; \cdot]\right.$ не изменяется при переходе к пространству $\left.S(\mathbb{R}, \mathscr{M})\right)$.

Так как $\mu[\cdot ; \cdot]=\sum_{l} \widetilde{\mu}_{l}[\cdot ; \cdot] \chi_{T_{l}}(\cdot)$ и $\left\{T_{l}\right\} \in \mathfrak{M}(\Lambda(\mu))$, то в силу леммы 1 имеем $\mu[\cdot ; \cdot] \in S(\mathbb{R}, \mathscr{M})$ и $\operatorname{Mod} \mu[\cdot ; \cdot] \subset \Lambda(\mu)$. Обратное включение $\Lambda(\mu) \subset \operatorname{Mod} \mu[\cdot ; \cdot]$ вытекает из следствия леммы 4. Теорема 1 доказана.

ЗАмечаниЕ 1 . Пусть $\delta_{x}$ - мера Дирака, сосредоточенная в точке $x \in \mathscr{U}$. Для любых $x, y \in \mathscr{U}$ справедливо равенство $d\left(\delta_{x}, \delta_{y}\right)=\theta(\rho(x, y))=\rho^{\prime}(x, y)$, из которого следует, что произвольная функция $f: \mathbb{R} \rightarrow \mathscr{U}$ принадлежит пространству $S(\mathbb{R}, \mathscr{U})$ тогда и только тогда, когда $\delta_{f(\cdot)} \in S(\mathbb{R}, \mathscr{M})$, при этом $\operatorname{Mod} f=\operatorname{Mod} \delta_{f(\cdot)}$. Поэтому частным случаем теоремы 1 (когда $\mu[\cdot ; t]=\delta_{f(t)}, t \in \mathbb{R}$ ) является следуюшее утверждение: Если $f: \mathbb{R} \rightarrow \mathscr{U}$ - произвольная функция в полном сепарабельном метрическом пространстве $\mathscr{U}$, то $f \in S(\mathbb{R}, \mathscr{U})$ в том и только том случае, если $\mathscr{F}(f(\cdot)) \in S_{1}$ для всех функций $\mathscr{F} \in C_{b}(\mathscr{U})$ при этом

$$
\operatorname{Mod} f=\sum_{\mathscr{F} \in C_{b}(\mathscr{U})} \operatorname{Mod} \mathscr{F}(f(\cdot)) .
$$


ЗАмЕчАнИЕ 2. Пусть $\mathscr{U}$ - полное сепарабельное метрическое пространство. Аналогично пространству $S(\mathbb{R}, \mathscr{M})$ можно рассмотреть пространство $B(\mathbb{R}, \mathscr{M})$ п.п. по Бору (равномерных п.п.) функций $[11],[12] t \rightarrow \mu[\cdot ; t], t \in \mathbb{R}$, со значениями в метрическом пространстве $(\mathscr{M}(\mathscr{U}), d)$. Если $\mu[\cdot ; \cdot] \in B(\mathbb{R}, \mathscr{M})$, то $\mu(\mathscr{F} ; \cdot) \in B$ для всех функций $\mathscr{F} \in C_{b}(\mathscr{U})$. Аналогично теореме 3 (с использованием теоремы 2 вместо ее следствия) доказывается утверждение: если в условиях теоремы $3 \mu(\mathscr{F} ; \cdot) \in B$ для всех $\mathscr{F} \in C_{b}(\mathscr{U})$, то $\left\|\mu\left(\mathscr{F}_{j} ; \cdot\right)\right\|_{B} \rightarrow 0$ при $j \rightarrow+\infty$. Из последнего утверждения вытекает аналог леммы 6 (при доказательстве которого не применяется лемма 3): если $\mu(\mathscr{F} ; \cdot) \in B$ для любой функции $\mathscr{F} \in C_{b}(\mathscr{U})$, то $\overline{\bigcup_{t \in \mathbb{R}} \mu[\cdot ; t]}-$ компакт в метрическом пространстве $\mathscr{M}$. Отсюда (как и при доказательстве теоремы 1 ), используя метрику $d^{0}$, получаем утверждение, аналогичное теореме $1:$ если $t \rightarrow \mu[\cdot ; t] \in \mathscr{M}, t \in \mathbb{R}$, - мерозначная функция такая, что $\mu(\mathscr{F} ; \cdot) \in B$ для всех $\mathscr{F} \in C_{b}(\mathscr{U})$, то $\mu[\cdot ; \cdot] \in B(\mathbb{R}, \mathscr{M})$ и

$$
\operatorname{Mod} \mu[\cdot ; \cdot]=\sum_{\mathscr{F} \in C_{b}(\mathscr{U})} \operatorname{Mod} \mu(\mathscr{F} ; \cdot) \text {. }
$$

Физико-технический институт

Поступило

Уральского отделения РАН

07.04 .95

\section{СПИСОК ЦИТИРОВАННОЙ ЛИТЕРАТУРЫ}

[1] Варга Дж. Оптимальное управление дифференциальными и функциональными уравнениями. М.: Наука, 1977.

[2] Красовский Н. Н. Управление динамической системой. М.: Наука, 1985.

[3] Ченцов А.Г. Приложение теории меры к задачам управления. Препринт. Свердловск, 1985.

[4] Иванов А. Г. Мерозначные почти периодические функции. Препринт. Свердловск, 1990.

[5] Иванов А. Г. О почти периодической ляпуновской задаче // ПММ. 1991. Т. 55. №5. C. $718-724$.

[6] Иванов А. Г. Оптимальное управление почти периодическими движениями // ПММ. 1992. T. 56. № 5. С. $133-142$.

[7] Данилов Л.И. О мерозначных почти периодических функциях // Вестн. Удмуртского ун-та. 1993. №1. С. 51-58.

[8] Данилов Л.И. Многозначные почти периодические отображения и их сечения // Деп. ВИНИТИ. № 2465-В93.

[9] Данилов Л. И., Иванов А. Г. К теореме опоточечном максимуме в почти периодическом случае // Изв. вузов. Матем. 1994. №6. С. 50-59.

[10] Данилов Л.И. О многозначных почти периодических отображениях, зависящих от параметра // Вестн. Удмуртского ун-та. 1994. № 2. С. 29-44.

[11] Левитан Б. М. Почти-периодические функции. М.: ГИТТЛ, 1953.

[12] Левитан Б. М., ЖКиков В. В. Почти периодические функции и дифференциальные уравнения. М.: Изд-во МГУ, 1978.

[13] Люстерник Л. А., Соболев В. И. Краткий курс функционального анализа. М.: Высшая шккола, 1982.

[14] Вахания Н.Н., Тариеладзе В. И., Чобанян С. А. Вероятностные распределения в банаховых пространствах. М.: Наука, 1985.

[15] Данилов Л.И. О суперпозиции почти периодических многозначных отображений и функций // Деп. ВИНИТИ. № 262-В95.

[16] Данилов Л. И. Почти периодические сечения многозначных отображений // Изв. отдела матем. и информ. УдГУ. 1993. №1. С. 16-78.

[17] Келли Дж. Л. Общая топология. М.: Наука, 1981. 